Reprod. Nutr. Dévelop., 1988, 28 Suppl. n 1, 177-178

\title{
La sécrétion des acides gras du lait en fonction de caractéristiques de la ration et de l'état nutritionnel chez la chèvre au démarrage de la lactation
}

D. SAUVANT, Nadine MANDRAN, J. HERVIEU, Françoise TERNOIS, Sylvie GIGER

Station de Nutrition et Alimentation

Institut national agronomique Paris-Grignon

16. rue C. Bernard, 75231 Paris Cedex 05

Summary. The amounts of 11 milk fatty acids secreted by 210 individual goats during the 2 nd week of lactation were explained by 4 variates : the digestible organic matter and ether extract intakes, the organic matter digestibility and a lipomobilization index. Significant effects varied according to the acids. About a quarter of the absorbed acetate equivalent appeared to be secreted as milkfat.

En raison de l'intérêt porté à la sécrétion lipidique du lait, il importe d'évaluer dans quelle mesure la production individuelle des acides gras par la mamelle peut être expliquée à l'aide de caractéristiques simples représentatives de leurs principales origines.

Matériel et méthodes. On a analysé les résultats obtenus en $2^{\mathrm{e}}$ semaine de lactation sur 210 chèvres réparties en 19 lots au cours de 6 expérimentations conduites de 1980 à 1986 . Toutes les rations contenaient du foin de luzerne, associé à de l'ensilage de maîs ou de la pulpe de betterave surpressée et à un aliment concentré distribué au prorata de la production. Dans chaque lot au moins un des aliments grossiers a été distribué à volonté. La digestibilité de la matière organique (DMO) des fourrages a été mesurée in vivo, les quantités d'aliments ingérés et de lait produit ont été mesurées individuellement et quotidiennement. La composition en acides gras a été mesurée dans un échantillon de lait représentatif d'une traite du soir et du matin. Après cette dernière, un prélèvement de sang jugulaire a été effectué pour doser les teneurs en acides gras non estérifiés (AGNE), $\beta$-hydroxybutyrate (BOHB) et glucose du plasma suivant les méthodes habituellement pratiquées au laboratoire.

Quatre caractères ont été utilisés dans une régression progressive ascendante pour expliquer les variations de la sécrétion des 11 principaux acides gras : la matière organique digestible ingérée (MODI) et l'extrait éthéré ingéré (EEI) exprimés en $\mathrm{g} / \mathrm{kgPV}^{0.75}$, la DMO, et un indice de lipomobilisation (IL) (Sauvant, Morand-Fehr et Bas 1984) constitué par une combinaison linéaire des teneurs en AGNE, $\beta-\mathrm{OHB}$ et glucose correspondant à la première composante principale extraite d'une analyse effectuée sur 804 triples de ces caractères mesurés en semaine 1, 2, 4 et 6 de lactation.

Résultats et discussion. Les quantités de MODI et EEI ont été respectivement de $67,0(\mathrm{~S}=11,4)$ et $2,8(\mathrm{~S}=1,1) \mathrm{g} / \mathrm{kgPV}^{0,75}$; elles sont significative- 
ment corrélées ( $R=0,44)$, mais ne sont pas liées avec l'indice de lipomobilisation; elles sont par contre significativement corrélées à la DMO (74,3, S = 2,6\%) du régime: $R=0,35$ et 0,27 respectivement.

L'indice IL est positivement associé aux quantités sécrétées de chacun des acides (tabl. 1). Son influence relative est plus marquée pour les acides à 18 atomes de carbone; elle diminue en outre progressivement de $4: 0$ à $12: 0$. Ce rôle positif de la lipomobilisation traduirait la fourniture d'acides gras longs par l'intermédiaire des $A G N E$, mais également celle des précurseurs courts issus de l'oxydation partielle des acides gras longs dans le foie. La quantité d'EEI influence significativement, avec IL, la production des acides $18: 0$ et $18: 1$. La quantité de MODI influence significativement la production des acides à moins de 18 atomes de carbone. Les coefficients de régression sont proportionnels aux quantités moyennes sécrétées de chaque acide. Ces valeurs cumulées indiquent qu'il y a 1,9 mmole d'équivalent acétate sécrété par ces acides par g de MODI. Sur la base des estimations de Thivend et Poncet (1978), il y aurait une absorption de 5,2 mmoles d'acétate, et de 1,2 mmole de butyrate par $\mathrm{g}$ de $\mathrm{MODI}$; avec cette hypothèse environ un quart du flux absorbé d'équivalent acétate serait sécrété sous forme d'acides gras de 4 à 16 atomes de $C$ dans le lait. En outre, pour les acides $4: 0$ à 12 : 0 , la sécrétion dépend significativement de la DMO du régime.

TABL. 1. - Niveau de sécrétion des acides gras du lait et résultats des calculs de régression.

\begin{tabular}{|c|c|c|c|c|c|c|c|}
\hline \multirow[b]{2}{*}{ Acide } & \multirow[b]{2}{*}{$\begin{array}{l}\text { Moyenne } \\
\text { (1) }\end{array}$} & \multirow[b]{2}{*}{$\begin{array}{c}\text { Ecart } \\
\text { type (1) }\end{array}$} & \multicolumn{5}{|c|}{ Coefficients de régression } \\
\hline & & & $\begin{array}{c}\text { MODI/ } / P^{0.75} \\
(2)\end{array}$ & DMO (\%) & IL & $\begin{array}{c}\mathrm{EEI} / \mathrm{P}^{0,75} \\
(2)\end{array}$ & $\mathrm{R}$ \\
\hline $4: 0$ & 146,4 & 71,0 & 1,6 & 12,5 & 23,6 & - & 0,71 \\
\hline $6: 0$ & 173,5 & 79,1 & 2,2 & 14,2 & 22,7 & - & 0,73 \\
\hline $8: 0$ & 210,7 & 86,1 & 2,8 & 12,9 & 23,5 & - & 0,71 \\
\hline $10: 0$ & 718,0 & 235,9 & 11,1 & 20,6 & 53,6 & - & 0,72 \\
\hline $12: 0$ & 335,4 & 108,4 & 5,0 & 11,1 & 10,2 & - & 0,67 \\
\hline $14: 0$ & 785,9 & 224,3 & 12,0 & - & 56,7 & - & 0.71 \\
\hline $16: 0$ & 2014,4 & 582,4 & 27,3 & - & 191,5 & - & 0,71 \\
\hline $16: 1$ & 157,2 & 72,0 & 1,3 & - & 20,0 & - & 0,63 \\
\hline $18: 0$ & 746,4 & 338,3 & - & - & 142,5 & 100,4 & 0,67 \\
\hline $18: 1$ & 1959,7 & 812,6 & - & - & 378,1 & 191,4 & 0,69 \\
\hline
\end{tabular}

(1) $\mathrm{mg} / \mathrm{j} ;$ (2) $\mathrm{g} / \mathrm{j}$

Malgré l'origine individuelle des observations, des combinaisons linéaires de caractères simples, représentatifs, des niveaux de disponibilité des précurseurs des lipides du lait permettent donc d'expliquer de façon très significative des niveaux de sécrétion des différents acides gras.

Sauvant D., Morand-Fehr P., Bas P., 1984. In Les maladies de la chèvre. Les Colloques de I'INRA $n^{\circ} 28$, pp. 369-378.

Thivend P., Poncet C., 1978. Communication personnelle à Vermorel, 1978. In INRA. L'Alimentation des ruminants. INRA Publication, 78000 Versailles. 